\title{
Design and Analysis of Genetically Constructed Logic Gates
}

\author{
S. Konur and M. Gheorghe \\ Department of Computer Science, Faculty of Engineering, University of Sheffield
}

\begin{abstract}
Synthetic biology, comprising many aspects including in vivo, in vitro and in silico techniques, models and methods, programming paradigms and tools, is a rapidly growing field with promising potential in building new synthetically constructed devices and systems. Synthetic biology features unconventional biological systems that do not naturally exist in nature. In this paper, we discuss a software platform, Infobiotics Workbench, developed to perform in silico experiments for synthetic biology systems. We utilise the tool on an unconventional system, a genetic logic gate.
\end{abstract}

Keywords Synthetic Biology; Genetic Logic Gates; Simulation; Verification

\section{INTRODUCTION}

Synthetic biology is a new field of biology allowing us to design and engineer new and unconventional biological systems. Such systems are inspired by natural processes occurring in biology or produce in vitro (DNA strands) or in vivo (bacteria) experiments simulating different computational devices. One specific class of models is the Boolean logic gates, a standard concept in computer science, which have been studied and designed unconventionally as genetic devices [1, 2].

In silico tools facilitate in vivo/vitro experiments by providing solutions for solving better or/and faster increasingly complex synthetic biology systems. In order to provide insight solutions, these tools should incorporate a set of methods for specifying, modelling, testing and simulating biological systems. In addition, they should provide support for algorithmic techniques, e.g. formal verification, to make analyses more comprehensive and discover novel information about system properties.

In this paper, we discuss the Infobiotics Workbench tool [3], a software platform developed to perform in silico experiments for synthetic biology systems. We utilise the tool on an unconventional system, a genetic logic gate.

\section{A SOFTWARE SUIT FOR SYNTHETIC BIOLOGY}

The Infobiotics Workbench (IBW) tool is a software environment for prototyping, designing and computationally analysing synthetic biology systems. IBW permits modelling, simulating, verifying and optimizing biological systems that exhibit molecular interactions.

In IBW, system models are described in a rule-based language, called stochastic $P(S P)$ systems [4], which facilitate the specification of multi-cellular systems and molecular interactions that take place in different locations of cells. The formalism also allows the modelling of membranes distributed geometrically.

IBW integrates various components utilised for dedicated tasks: the model editor component allows the construction and editing biological models described as SP system models; the stochastic simulation component permits carrying out deterministic and stochastic simulations to observe the dynamics of the system analysed; the verification component provides an exhaustive analysis of the system in question (by encoding the system into a formal system) to infer more novel information about the system properties (which cannot be normally obtained using simulation); the optimization component allows estimating and/or optimizing system parameters and the dashboard component is responsible for displaying results in various formats, e.g. time series, bars, histograms, 2D/3D heat-map meshes or surfaces and 3D animations.

\section{A BASIC Genetic Logic Gate}

In synthetic biology, logic gates have been genetically designed and constructed in several papers, including [1, 2]. In this paper, we model a genetic AND gate using the IBW tool, and computationally analyse the model to confirm that it works like an ordinary logic gate.

Fig. 1 illustrates the truth table, the logic diagram and the genetic device functioning as an AND gate. The parts used in this device are the same genetic parts used in [1]. The device receives two inputs: the aTc and IPTG molecules. aTc (resp. IPTG) disables the activity of the transcription factor TetR (resp. Lacl). A gene controlled by the same promoter synthesises Lacl and TetR. aTc (resp. IPTG) inhibits TetR (resp. Lacl) to prevent it from repressing the production of GFP by binding to the corresponding promoter upregulating the expression of GFP. If both IPTG and aTc are set to high, then neither Lacl nor TetR can inhibit the GFP production.

\section{ANALYSIS}

The IBW suit permits various computational analysis in order to observe the behaviour of a system. Here, we present the simulation and verification results performed using IBW's corresponding components. 


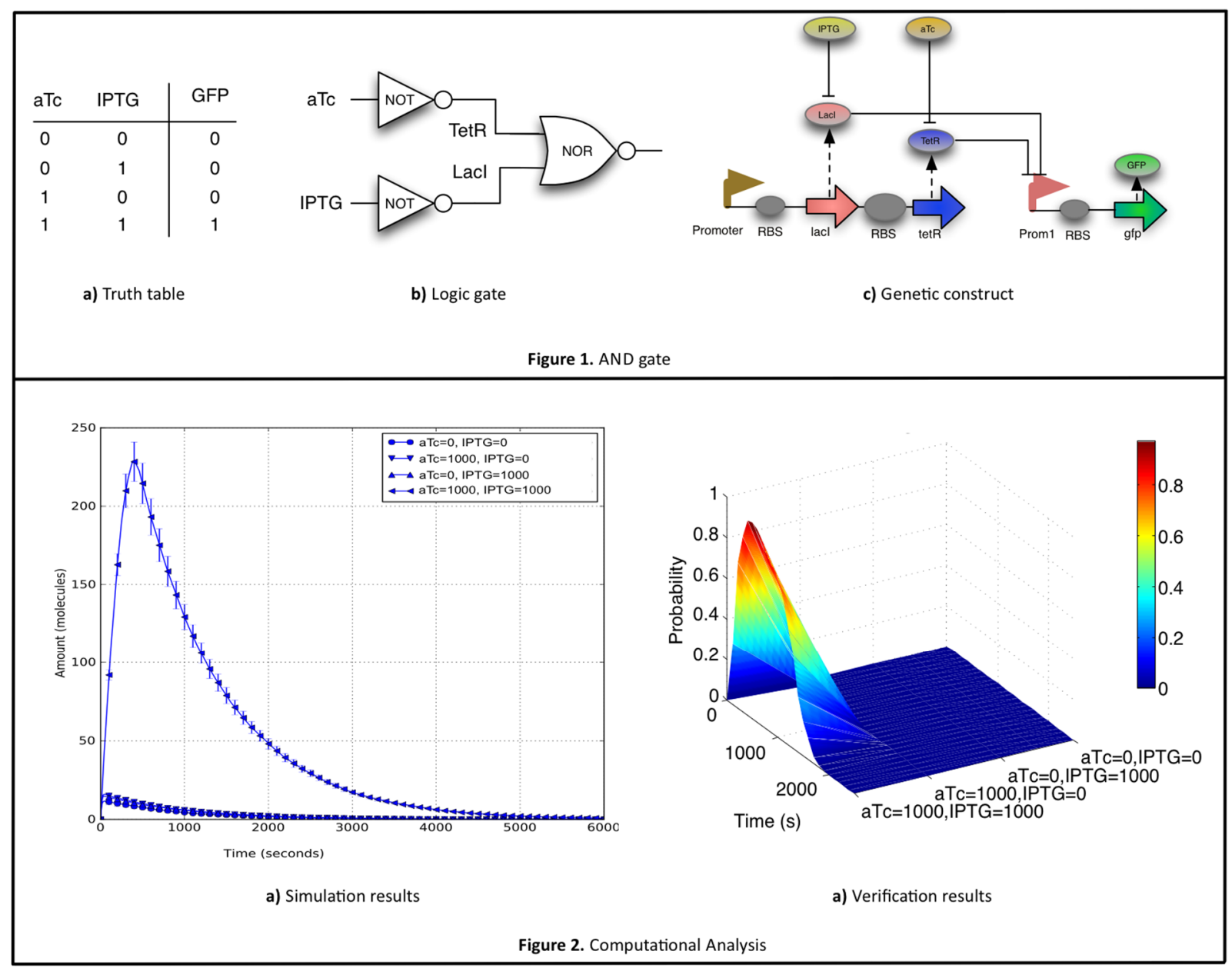

Fig. 2(a) presents the GFP amount varying over time. As shown in the figure, GFP is observed in high concentration only if both the aTc and IPTG molecules are set to high, confirming Fig. 1(a).

To formally verify a system, IBW automatically constructs the corresponding (mathematical) formal model, which can be queried using a dedicated logical formalism. To assist non-experts, IBW also features a tool translating queries expressed in natural language into their formal logical syntax. For the AND gate, we have verified the following query:

"What is the probability that GFP exceeds Thr at time $t$ ?"

In Fig. 2(b) presents the verification results; namely, the verification results for a threshold value of 100 over the time points up to 2500 seconds. The results clearly confirm the desired behaviour.

\section{Conclusions}

In this paper, we have presented how the IBW tool utilises the design and analysis of synthetic biological devices and systems using different computational techniques, e.g. simulation and verification. We have also presented our initial results on a basic genetic AND device.

We are currently working on a new version of IBW, which will incorporate a set of methods for specifying, modelling, testing and simulating biological systems, and will facilitate these processes for biologists. In addition, the version will also include a biocompilation module that will combine genetic parts databases, and user knowledge, to automatically build a viable DNA sequence that can be used in organisms.

\section{ACKNOWLEDGEMENTS}

The authors acknowledge the support provided for synthetic biology research by EPSRC ROADBLOCK project (EP/I031812/1).

\section{REFERENCES}

1. J. Beal, A. Phillips, D. Densmore, and Y. Cai. High-level Programming Languages for Biomolecular Systems. 2011.

2. Tamsir, J. J. Tabor, and C. A. Voigt. Robust Multicellular Computing using Genetically Encoded NOR Gates and Chemical 'Wires'. 2011.

3. J. Blakes, J. Twycross, S. Konur, F. J. Romero-Campero, N. Krasnogor, and M. Gheorghe. Infobiotics Workbench: A P Systems based Tool for Systems and Synthetic Biology. 2014.

4. F. J. Romero-Campero, J. Twycross, H. Cao, J. Blakes, and N. Krasnogor. A Multiscale Modeling Framework based on $\mathrm{P}$ Systems. 2009. 